\title{
RESTORATION OF DEGRADED MOVING IMAGE FOR PREDICTING A MOVING OBJECT
}

\author{
Kei Akiyama ${ }^{1), 2)}$, Zhi-wei Luo $^{2)}$, Masaki Onishi $^{2)}$ and Shigeyuki Hosoe ${ }^{l), 2)}$ \\ 1) Graduate School of Engineering, Nagoya University \\ Furo-cho, Chikusa-ku Nagoya, 464-8603 JAPAN \\ Email: \{k_akiyama,hosoe\}@nuem.nagoya-u.ac.jp \\ 2) Bio-mimetic Control Research Center, RIKEN \\ 2271-130, Anagahora, Shimoshidami, Moriyama-ku Nagoya, 463-0003 JAPAN \\ Email: \{luo,onishi\}@bmc.riken.jp
}

\begin{abstract}
Keywords: Moving image restoration, wavelet multiresolution analysis, nonlinear optimization, dynamics of moving image.

Abstract: $\quad$ Iterative optimal calculation methods have been proposed for restoration of degraded static image based on wavelet multiresolution decomposition. However, it is quite difficult to apply these methods to process moving images due to the high computation cost. In this paper, we propose an effective restoration method for degraded moving image by modeling the motion of a moving object and predicting the future object position. We verified our method by computer simulations and an experiment to show that our method can reduce the computation time.
\end{abstract}

\section{INTRODUCTION}

When measuring external world by a camera, degradation in the observed images may be caused by many factors. It is then important to estimate the original image and to restore the observed one. By now, in the research field of computer vision, there are several approaches be proposed for degraded image restoration (Geman and Yang, 1995; Osher et al., 1992; Belge et al., 2000). One of these approaches (Belge et al., 2000) even enables the edge-preserving restoration of an image by using wavelet multiresolution decomposition (Mallat, 1989). However, because of huge amount of computation cost, this approach can only be applied for the restoration of a static image but is ineffective to process the moving image.

In this paper, we propose a novel restoration method for a moving image by developing the Belge et al.'s approach. In our algorithm, we model the dynamics of moving image and calculate the restored image using the predicted image that is calculated based on the dynamic model of the moving object. We verify our method by computer simulation of artificially generated moving image and an experiment of a real moving image, which show that our method can realize image restoration while reducing computation time.

\section{STATIC IMAGE RESTORATION}

In this section, we first review the restoration method for a static image (Belge et al., 2000) using wavelet multiresolution decomposition.

A general degradation process of an $N \times N$ static image can be formulated as

$$
g=H f+u
$$

where the vectors $g, f$ and $\boldsymbol{u}$ represent the lexicographically ordered degraded image, the original image and the disturbance, respectively. The matrix $\boldsymbol{H}$ represents optical blur or linear distortion. With 2-D wavelet multiresolution (Mallat, 1989), equation (1) can be converted into the following form

$$
\hat{g}=\hat{H} \hat{f}+\hat{u}
$$

where $\hat{\boldsymbol{g}}, \hat{\boldsymbol{H}}, \hat{\boldsymbol{f}}, \hat{\boldsymbol{u}}$ are $\hat{\boldsymbol{g}}=\mathcal{W} \boldsymbol{g}, \hat{\boldsymbol{f}}=\mathcal{W} \boldsymbol{f}, \hat{\boldsymbol{u}}=\mathcal{W} \boldsymbol{u}$ and $\hat{\boldsymbol{H}}=\mathcal{W} \boldsymbol{H} \mathcal{W}^{T}$ with a wavelet multiresolution decomposition matrix $\mathcal{W}$, respectively. $\mathcal{W}$ is orthogonal, that is $\mathcal{W}^{T} \mathcal{W}=\boldsymbol{I}$.

Wavelet multiresolution decomposition is a method of decomposing an image into multiresolution images by utilizing orthogonal wavelet transformation. A block diagram of two level wavelet decomposition of image $\boldsymbol{f}$ is shown in Fig. 1, in which $p(\cdot)$ and $q(\cdot)$ are 1-D low-pass and high-pass filters. From the input image, four down-sampled images (LL, HL, LH, HH) 


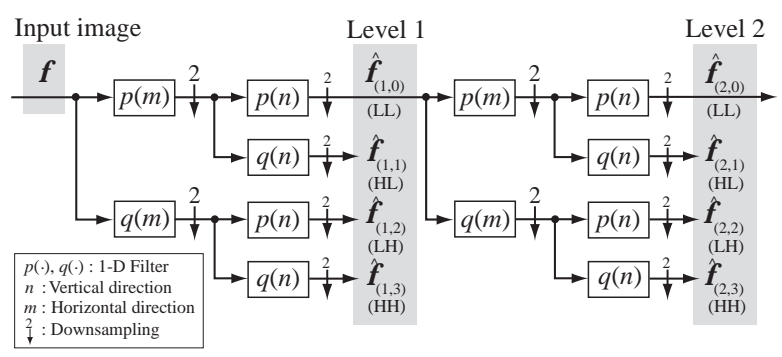

Figure 1: Two level multiresolution wavelet decomposition of an image $f$.

are obtained. Furthermore, by repeating the decomposition, we can get multiresolution images. Note that the number of the total pixels is unchanged during the decomposition.

The optimal restored image for the degradation process (2) is obtained by minimizing the next cost function about $\hat{f}$ (Belge et al., 2000)

$$
\begin{aligned}
J(\hat{\boldsymbol{f}}, \boldsymbol{\lambda})= & \|\hat{\boldsymbol{g}}-\hat{\boldsymbol{H}} \hat{\boldsymbol{f}}\|_{2}^{2}+\lambda_{(L, 0)}\left\|\hat{\boldsymbol{f}}_{(L, 0)}\right\|_{p}^{p} \\
& +\sum_{l=1}^{L} \sum_{j=1}^{3} \lambda_{(l, j)}\left\|\hat{\boldsymbol{f}}_{(l, j)}\right\|_{p}^{p} .
\end{aligned}
$$

The subscript $l$ and $j$ denote the decomposition level and the type of the decomposed image, respectively. The optimal restored image can be obtained by calculating $\hat{f}^{*}$ such that the differentiation of the cost function (3) approaches to 0 . Then we get

$$
\begin{aligned}
& \left(\hat{\boldsymbol{H}}^{T} \hat{\boldsymbol{H}}+\frac{p}{2} \boldsymbol{D}^{*}\right) \hat{\boldsymbol{f}}^{*}=\hat{\boldsymbol{H}}^{T} \hat{\boldsymbol{g}} \\
& \boldsymbol{D}^{*}=\operatorname{diag}\left[\frac{\boldsymbol{\lambda}(i)}{\left(\left|\hat{\boldsymbol{f}}^{*}(i)\right|^{2}+\beta\right)^{1-p / 2}}\right]_{i=1}^{N^{2}}
\end{aligned}
$$

(Belge et al., 2000). Here, $\hat{\boldsymbol{f}}^{*}(i)$ denotes the $i$ th element of $\hat{\boldsymbol{f}}^{*}, \boldsymbol{\lambda}(i)$ is the weight corresponds to $\hat{\boldsymbol{f}}^{*}(i)$, and $\beta(\geq 0)$ is the stabilization constant for approximation of $L_{p}$ norm terms (Belge et al., 2000), respectively.

To solve this nonlinear equation, an iterative calculation method can be applied as follows (Belge et al., 2000)

$$
\begin{aligned}
& \left(\hat{\boldsymbol{H}}^{T} \hat{\boldsymbol{H}}+\frac{p}{2} \boldsymbol{D}^{(k)}\right) \hat{\boldsymbol{f}}^{(k+1)}=\hat{\boldsymbol{H}}^{T} \hat{\boldsymbol{g}} \\
& \boldsymbol{D}^{(k)}=\operatorname{diag}\left[\frac{\boldsymbol{\lambda}(i)}{\left(\left|\hat{\boldsymbol{f}}^{(k)}(i)\right|^{2}+\beta\right)^{1-p / 2}}\right]_{i=1}^{N^{2}}
\end{aligned}
$$

where the superscript $(k)$ expresses an iteration number of times. If $p \geq 1$ and $\hat{\boldsymbol{H}}$ is full rank, it is shown that the iterative calculation by (6) and (7) converges to the solution of the nonlinear equations (4) and (5) $\left(\hat{\boldsymbol{f}}^{*}\right)$ with a suitable initial value $\hat{\boldsymbol{f}}^{(0)}$ when $k \rightarrow \infty$ (Charbonnier et al., 1997).

The above method can remove the degradation factors while preserving local patterns of an image by assigning different weight $\lambda_{(l, j)}$ to each decomposed image. Here, $\boldsymbol{\lambda}$ is set as a constant for simplicity.

\section{MOVING IMAGE RESTORATION}

\subsection{Application to the Moving Image Restoration}

In this paper, we consider the moving image restoration problem to restore a time series of original images $\left(\boldsymbol{f}^{[1]}, \cdots, \boldsymbol{f}^{[K]}\right)$ from a degraded time series of observed images $\left(\boldsymbol{g}^{[1]}, \cdots, \boldsymbol{g}^{[K]}\right)$. Here, it is noted that the superscript $[k]$ expresses a frame number of a moving image whereas the superscript $(k)$ in equations (6) and (7) expressed the iteration number of times.

We suppose that a degradation process of an image is similar to the case of static image:

$$
\boldsymbol{g}^{[k]}=\boldsymbol{H} \boldsymbol{f}^{[k]}+\boldsymbol{u}^{[k]}, \quad k=1, \cdots, K .
$$

In this work, we assume $\boldsymbol{H}$ to be constant, that is, the optical blur or the distortion is independent of each frame.

Since equations (6) and (7) represent iterative calculation with a huge $\left(\operatorname{order}\left(N^{2}\right)\right)$ matrix, it requires high computational cost when applying this algorithm directly in our moving image restoration. Therefore, we assume the following properties about the original moving image and propose our approach to reduce the calculation cost.

\section{Assumption about an original image}

1. An original moving image consists of a foreground and a background.

2. The change of the background is so small so as be set as a static image.

3. The change of the foreground can be formulated or be approximated by known dynamics such as linear or parabolic movements as will be mentioned later.

4. The foreground is assumed to be a single rigid body with smoothly changing pixel value in the domain and maintain its orientation.

When the assumptions 1 and 2 hold, we can utilize the restoration result of previous frame directly as an initial estimation of the background for each frame. On the other hand, we can predict a new position of the foreground from the previous restoration result and the information about motion dynamics (assumption 


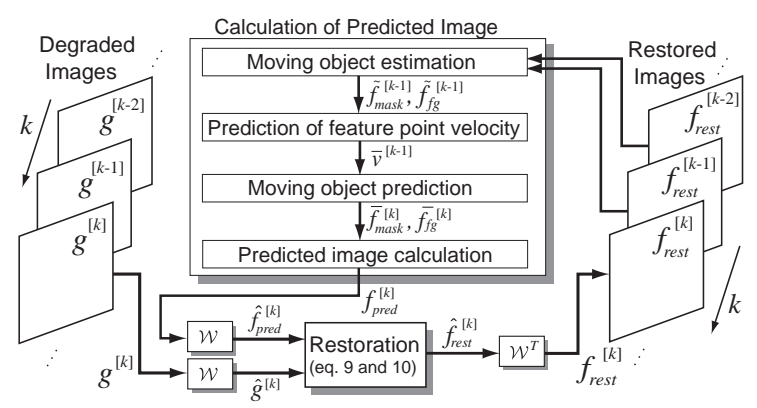

Figure 2: Overview of the proposed method at $k$ th frame.

3) by using Kalman filter. Therefore, by using these images as an initial value in the equations (6) and (7), we can show that good restoration results can be obtained by only one time restoration calculation, in section 4. In addition, for the moving image which stood static, our algorithm agrees with the Belge et al.'s algorithm.

Based on the above description, we modify the foregoing iterative calculation (6) and (7) as follows:

$$
\begin{gathered}
\left(\hat{\boldsymbol{H}}^{T} \hat{\boldsymbol{H}}+\frac{p}{2} \boldsymbol{D}_{\text {pred }}^{[k]}\right) \hat{\boldsymbol{f}}_{\text {rest }}^{[k]}=\hat{\boldsymbol{H}}^{T} \hat{\boldsymbol{g}}^{[k]} \\
\boldsymbol{D}_{\text {pred }}^{[k]}=\operatorname{diag}\left[\frac{\boldsymbol{\lambda}(i)}{\left(\left|\hat{\boldsymbol{f}}_{\text {pred }}^{[k]}(i)\right|^{2}+\beta\right)^{1-p / 2}}\right]_{i=1}^{N^{2}} .
\end{gathered}
$$

Here, $\hat{\boldsymbol{f}}_{\text {rest }}^{[k]}$ is a restored image of $k$ th frame in the wavelet domain, and $\hat{\boldsymbol{f}}_{\text {pred }}^{[k]}$ is a predicted image of $k$ th frame. We denote a restored and a predicted images in space domain as $\boldsymbol{f}_{\text {rest }}^{[k]}$ and $\boldsymbol{f}_{\text {pred }}^{[k]}$. A summary of our proposed method is shown in Fig. 2. The dynamics of the moving image will be formulated later and will be used to calculate the predicted image $\hat{\boldsymbol{f}}_{p r e d}^{[k]}$. For a degraded image $\hat{\boldsymbol{g}}^{[k]}$, we calculate a restored image $\boldsymbol{f}_{\text {rest }}^{[k]}$ by equations (9) and (10) using $\hat{\boldsymbol{f}}_{\text {pred }}^{[k]}$.

\subsection{Formulation Dynamics of Moving Image}

Based on the above assumptions, we model dynamics of an original moving image as follows. At first we define each variables.
$\boldsymbol{f}^{[k]} \quad$ FOriginal image vector of $k$ th frame
$\boldsymbol{f}_{\text {mask }}^{[k]}$ FOriginal moving object domain vector
$\boldsymbol{f}_{b g} \quad$ FOriginal background image vector
$\boldsymbol{f}_{f g}^{[k]} \quad$ FOriginal foreground image vector

Original moving object domain vector is a vector in which pixels where the background is covered behind the moving object are 0 , and the others are 1 . Original foreground image vector is made by turned over with
0 and 1 of $\boldsymbol{f}_{\text {mask }}^{[k]}$, and multiplied by the original pixel value of the moving object. All the vectors are with $N^{2}$ dimension. By the definitions, they satisfy

$$
\boldsymbol{f}^{[k]}=\operatorname{diag}\left(\boldsymbol{f}_{b g}(1), \ldots, \boldsymbol{f}_{b g}\left(N^{2}\right)\right) \cdot \boldsymbol{f}_{\text {mask }}^{[k]}+\boldsymbol{f}_{f g}^{[k]} \text {. }
$$

For transition of moving object domain $\boldsymbol{f}_{\text {mask }}^{[k]}$ with constant acceleration for an example, we get

$$
\begin{aligned}
\boldsymbol{f}_{\text {mask }}^{[k+1]} & =\boldsymbol{T}\left(\boldsymbol{v}^{[k]}\right) \boldsymbol{f}_{\text {mask }}^{[k]} \\
{\left[\begin{array}{c}
\boldsymbol{v}^{[k+1]} \\
\boldsymbol{a}^{[k+1]}
\end{array}\right] } & =\left[\begin{array}{ll}
\boldsymbol{I} & \boldsymbol{I} \\
\mathbf{0} & \boldsymbol{I}
\end{array}\right]\left[\begin{array}{c}
\boldsymbol{v}^{[k]} \\
\boldsymbol{a}^{[k]}
\end{array}\right] .
\end{aligned}
$$

We call $\boldsymbol{T}\left(\boldsymbol{v}^{[k]}\right)$ a transition matrix. Vectors $\boldsymbol{v}^{[k]}=$ $\left(v_{x}^{[k]}, v_{y}^{[k]}\right)^{T}$ and $\boldsymbol{a}^{[k]}=\left(a_{x}^{[k]}, a_{y}^{[k]}\right)^{T}$ are velocity and acceleration of the moving object, and subscripts $x, y$ express vertical and horizontal directions. From the assumption 4, we can regard transition of an moving object as a translation. Therefore, transition matrix can be expressed as follows:

$$
\boldsymbol{T}\left(\boldsymbol{v}^{[k]}\right)=\operatorname{diag}\left(\boldsymbol{C}_{y}{ }^{v_{y}^{[k]}}, \ldots, \boldsymbol{C}_{y}^{v_{y}^{[k]}}\right) \cdot \boldsymbol{C}_{x}^{v_{x}^{[k]}} .
$$

Matrix $C_{x}$ and $C_{y}$ in equation (14) are following $N^{2} \times N^{2}$ dimension block circulant matrix and $N \times N$ dimension circulant matrix:

$$
\begin{aligned}
\boldsymbol{C}_{x}= & {\left[\begin{array}{cccccc}
\mathbf{0} & \mathbf{0} & \mathbf{0} & \cdots & \mathbf{0} & \boldsymbol{I} \\
\boldsymbol{I} & \mathbf{0} & \mathbf{0} & \cdots & \mathbf{0} & \mathbf{0} \\
\mathbf{0} & \boldsymbol{I} & \mathbf{0} & \cdots & \mathbf{0} & \mathbf{0} \\
\vdots & \ddots & \ddots & \ddots & \ddots & \vdots \\
\mathbf{0} & \cdots & \mathbf{0} & \boldsymbol{I} & \mathbf{0} & \mathbf{0} \\
\mathbf{0} & \cdots & \mathbf{0} & \mathbf{0} & \boldsymbol{I} & \mathbf{0}
\end{array}\right] } \\
\boldsymbol{C}_{y}= & {\left[\begin{array}{cccccc}
0 & 0 & 0 & \cdots & 0 & 1 \\
1 & 0 & 0 & \cdots & 0 & 0 \\
0 & 1 & 0 & \cdots & 0 & 0 \\
\vdots & \ddots & \ddots & \ddots & \ddots & \vdots \\
0 & \cdots & 0 & 1 & 0 & 0 \\
0 & \cdots & 0 & 0 & 1 & 0
\end{array}\right] . }
\end{aligned}
$$

Here, we express them with

$$
\boldsymbol{C}_{x}^{-v_{x}^{\prime[k]}}:=\left(\boldsymbol{C}_{x}^{-1}\right)^{v_{x}^{\prime[k]}}, \boldsymbol{C}_{y}^{-v_{y}^{\prime[k]}}:=\left(\boldsymbol{C}_{y}{ }^{-1}\right)^{v_{y}^{\prime[k]}}
$$

in the case of $v_{x}^{[k]}=-v_{x}^{\prime[k]}<0$ or $v_{y}^{[k]}=-v_{y}^{\prime[k]}<0$. In addition, the transition of foreground image $\boldsymbol{f}_{f g}^{[k+1]}$ is provided by the same transition matrix $\boldsymbol{T}\left(\boldsymbol{v}^{[k]}\right)$.

Moreover, we can describe the rotation or the expansion / reduction of an moving object domain by modeling the transition matrix.

\subsection{Algorithm for Moving Image Restoration}

The moving image restoration algorithm is given as follows: 
Moving image restoration algorithm in $K$ frames

1. Give a predicted image $\hat{f}_{\text {pred }}^{[1]}$ when $k=1$, properly.

2. Calculate the restored image $\hat{\boldsymbol{f}}_{\text {rest }}^{[k]}$ by equations (9) and (10) with $\hat{\boldsymbol{f}}_{p r e d}^{[k]}$ for the degraded image $\hat{\boldsymbol{g}}^{[k]}$. Calculate $\boldsymbol{f}_{\text {rest }}^{[k]}=\mathcal{W}^{T} \hat{\boldsymbol{f}}_{\text {rest }}^{[k]}$.

3. Calculate an estimation of a moving object domain $\left(\tilde{\boldsymbol{f}}_{\text {mask }}^{[k]}\right)$ from $\boldsymbol{f}_{\text {rest }}^{[k]}$.

4. With $\tilde{\boldsymbol{f}}_{\text {mask }}^{[k]}$, divide $\boldsymbol{f}_{\text {rest }}^{[k]}$ into the estimated foreground $\left(\tilde{\boldsymbol{f}}_{f g}^{[k]}\right)$ and background $\left(\tilde{\boldsymbol{f}}_{b g}^{[k]}\right)$. For each pixel, if $\tilde{\boldsymbol{f}}_{\text {mask }}^{[k]}[(n-1) N+m]=1$, then

$\tilde{\boldsymbol{f}}_{f g}^{[k]}[(n-1) N+m]:=0$, and

$\tilde{\boldsymbol{f}}_{b g}^{[k]}[(n-1) N+m]:=\boldsymbol{f}_{\text {rest }}^{[k]}[(n-1) N+m]$.

Else, if $\tilde{\boldsymbol{f}}_{\text {mask }}^{[k]}[(n-1) N+m]=0$, then

$\tilde{\boldsymbol{f}}_{f g}^{[k]}[(n-1) N+m]:=\boldsymbol{f}_{\text {rest }}^{[k]}[(n-1) N+m]$, and

$\tilde{\boldsymbol{f}}_{b g}^{[k]}[(n-1) N+m]:=0$,

$(n=1, \cdots, N, m=1, \cdots, N)$.

5. Detect a certain characteristic point $\boldsymbol{c}^{[k]}=$ $\left(c_{x}^{[k]}, c_{y}^{[k]}\right)^{T}$ (center of gravity, for example) of $\tilde{\boldsymbol{f}}_{f g}^{[k]}$, and calculate a predicted value $\left(\overline{\boldsymbol{c}}^{[k+1]}\right)$ in $k+1$ th frame by Kalman filter. Calculate a predicted velocity $\left(\overline{\boldsymbol{v}}^{[k]}\right)$ and the transition matrix $\boldsymbol{T}\left(\overline{\boldsymbol{v}}^{[k]}\right)$ successively. Calculate a predicted moving object domain $\left(\overline{\boldsymbol{f}}_{\text {mask }}^{[k+1]}\right)$ by:

$$
\overline{\boldsymbol{f}}_{\text {mask }}^{[k+1]}:=\boldsymbol{T}\left(\overline{\boldsymbol{v}}^{[k]}\right) \tilde{\boldsymbol{f}}_{\text {mask }}^{[k]} .
$$

6. Calculate the predicted foreground image $\left(\overline{\boldsymbol{f}}_{f g}^{[k+1]}\right)$ using the transition matrix $\boldsymbol{T}\left(\overline{\boldsymbol{v}}^{[k]}\right)$ and $\tilde{\boldsymbol{f}}_{f g}^{[k]}$. Calculate the predicted background image $\left(\bar{f}_{b g}^{[k+1]}\right)$ by taking average of $\tilde{\boldsymbol{f}}_{b g}^{[l]}(l=1, \cdots, k)$ for each pixel.

7. With $\overline{\boldsymbol{f}}_{m a s k}^{[k+1]}, \overline{\boldsymbol{f}}_{f g}^{[k+1]}$ and $\overline{\boldsymbol{f}}_{b g}^{[k+1]}$ provided by steps 5 and 6 , calculate the predicted image $f_{\text {pred }}^{[k+1]}$ by the next expression corresponding to equation (11).

$$
\begin{array}{r}
\boldsymbol{f}_{\text {pred }}^{[k+1]}:=\operatorname{diag}\left(\overline{\boldsymbol{f}}_{b g}^{[k+1]}(1), \ldots, \overline{\boldsymbol{f}}_{b g}^{[k+1]}\left(N^{2}\right)\right) \\
\cdot \overline{\boldsymbol{f}}_{\text {mask }}^{[k+1]}+\overline{\boldsymbol{f}}_{f g}^{[k+1]}
\end{array}
$$

Calculate $\hat{\boldsymbol{f}}_{\text {pred }}^{[k+1]}=\mathcal{W} \boldsymbol{f}_{\text {pred }}^{[k+1]}$.

8. Repeat the steps 2 to 7 for $k=1, \cdots, K$. Terminate at step 2 for $k=K$.

In addition, if we could not predict it for the reasons of frame-out of the moving object or an change of the
Table 1: Parameters used in the simulation.

\begin{tabular}{c||c}
\hline \hline item name & value \\
\hline Optical blur $\left(\sigma^{2}\right)$ & 1.7 \\
SN ratio of the disturbance & $15 \mathrm{~dB}$ \\
Level of the decomposition $(L)$ & 3 \\
$\left(\lambda_{1}, \lambda_{2}\right)$ & $(0.1,0.3)$ \\
$p$ & 1.0 \\
$\alpha$ & 1.2 \\
$\beta$ & $10^{-2}$ \\
\hline
\end{tabular}

scene, we cancel the prediction till the next moving object is observed.

Considering the limited paper length we eliminated the detailed calculations in steps 3 and 5 .

\section{SIMULATION STUDIES}

We performed a simulation of the proposed method for an artificially generated and a real moving images. The artificial moving image has known degradation parameters and we verified the performance of the proposed method quantitatively. The degradation parameters of the real moving image are unknown, so we verified it qualitatively.

\subsection{Restoration Simulation of an Artificial Moving Image}

We generated an artificial moving image in $128 \times 128$ pixels and 36 frames. We used a test image LAX for the background and an arbitrary-shaped object with uniform pixel value for the foreground. The foreground was supposed to move with constant velocity. We made the original moving image $f^{[k]}$ by equation (11) and calculated its degraded moving image $\boldsymbol{g}^{[k]}$ by equation (8). In addition, we considered an optical blur for $\boldsymbol{H}$ in equation (8) and used a Gauss function of the variance $\sigma^{2}=1.7$ with the $9 \times 9$ discretized elements. In addition, the disturbance $\boldsymbol{u}^{[k]}$ was assumed to be a Gaussian noise of average of 0 and SN ratio of $15 \mathrm{~dB}$ independent between each frames. In the restoration calculation, the level of the wavelet multiresolution decomposition $(L)$ was assumed to be 3 and used the three tap wavelet (Daubechies, 1992). Besides, the parameter $\lambda_{(l, j)}$ was set as follows (Belge et al., 2000):

$$
\begin{gathered}
\lambda_{(3,0)}=\lambda_{1}, \quad \lambda_{(l, j)}=\lambda_{2} 2^{-\alpha(l-3)} \\
(l=1,2, j=1,2,3)
\end{gathered}
$$

The parameters we used in the simulation are given in Table 1. The predicted image in step 1 of the proposed algorithm was assumed to be $\hat{\boldsymbol{f}}_{\text {pred }}^{[1]}=\hat{\boldsymbol{g}}^{[1]}$. In addition, we did not calculate predicted images and 
Table 2: Comparison of the simulation result of 32 nd frame.

\begin{tabular}{c||c|c|c|c}
\hline \hline item name & Proposed & Compared 1 & Compared 2 & (Belge et al., 2000) \\
\hline Cost value of a restored image $\left(\times 10^{5}\right)$ & 1.54 & 1.52 & 1.52 & 1.50 \\
Cost value of an initial image $\left(\times 10^{5}\right)$ & 1.85 & 5.15 & 3.96 & 3.96 \\
ISNR[dB] & 3.27 & 3.25 & 3.14 & 2.54 \\
Iterative calculation number of times & 1 & 2 & 2 & 30 \\
Calculation time & $28^{\prime \prime}$ & $5{ }^{\prime} 20^{\prime}$ & $50^{\prime \prime}$ & $1: 14^{\prime} 17^{\prime \prime}$ \\
Prediction time (of which calc. time) & $1{ }^{\prime \prime}$ & - & - & - \\
\hline
\end{tabular}

just set $\hat{\boldsymbol{f}}_{\text {pred }}^{[k+1]}:=\hat{\boldsymbol{f}}_{\text {rest }}^{[k]}$ in $k=1,2$ and 3 because there existed a big change in the restored images of these frames. We calculated the predicted images in the other frames.

We compared our approach with the following two cases.

- With the restored image of previous frame for the initial value, iterate calculation of equations (6) and (7) till the cost value of a restored image in the proposed method is provided in each frame (compared method 1).

- With the current degraded image for the initial value, iterate calculation of equations (6) and (7) till the cost value of a restored image in the proposed method is provided in each frame (compared method 2).

\subsubsection{Simulation Result for One Representative Frame}

We compare a restoration result of 32nd frame as an example here. We show numerical values of the restored images of each method in Table 2. Moreover, for reference, we also show numerical values of a restoration result of the method in which

- With the current degraded image for the initial value, iterate calculation of equations (6) and (7) till it converges in each frame (Belge et al.'s method)

in the table. Here, we judged the restored image of $k^{\prime}$ th iteration number of times of 32 nd frame $\left(\hat{\boldsymbol{f}}_{\text {rest }}^{[32]\left(k^{\prime}\right)}\right)$ to have converged when

$\left\|\hat{\boldsymbol{f}}_{\text {rest }}^{[32]\left(k^{\prime}\right)}-\hat{\boldsymbol{f}}_{\text {rest }}^{[32]\left(k^{\prime}-1\right)}\right\| /\left\|\hat{\boldsymbol{f}}_{\text {rest }}^{[32]\left(k^{\prime}-1\right)}\right\|<5.0 \times 10^{-4}$ and broke off the calculation (Belge et al., 2000).

In the first and second lines of the table, we show the cost values which were calculated for a restored image or an initial image by the following cost function

$$
\begin{aligned}
J\left(\hat{\boldsymbol{\eta}}^{[k]}, \boldsymbol{\lambda}\right)= & \left\|\hat{\boldsymbol{g}}^{[k]}-\hat{\boldsymbol{H}} \hat{\boldsymbol{\eta}}^{[k]}\right\|_{2}^{2}+\lambda_{(L, 0)}\left\|\hat{\boldsymbol{\eta}}_{(L, 0)}^{[k]}\right\|_{p}^{p} \\
& +\sum_{l=1}^{L} \sum_{j=1}^{3} \lambda_{(l, j)}\left\|\hat{\boldsymbol{\eta}}_{(l, j)}^{[k]}\right\|_{p}^{p}
\end{aligned}
$$

corresponding to equation (3). In the second line, an initial image of each method corresponds to the predicted image (proposed method), the restored image of previous frame (compared method 1) and the current degraded image (compared method 2 and the Belge et al.'s method). The cost value of the predicted image of the proposed method is smaller than those of the initial images of the compared method 1 and 2 .

In the third line, we show ISNR (Improved Signal to Noise Ratio) (Banham and Katsaggelos, 1997) calculated by the next equation:

$$
\text { ISNR }=10 \log _{10} \frac{\left\|\boldsymbol{g}^{[k]}-\boldsymbol{f}^{[k]}\right\|^{2}}{\left\|\boldsymbol{f}_{\text {rest }}^{[k]}-\boldsymbol{f}^{[k]}\right\|^{2}}[\mathrm{~dB}] .
$$

ISNR of the proposed method is $3.27 \mathrm{~dB}$, which is an enough good restoration result. In addition, ISNR of the proposed method is similar as a result of the compared method 1, and be better than that of the compared method 2. On the other hand, ISNR of the Belge et al.'s method is smaller, however, it is good from the subjective evaluation as will be mentioned later. Such a tendency can be seen in other frames.

Furthermore, we show the iterative calculation number of times of each method in the fourth line. In the fifth line, we show the each calculation time. The prediction time in our proposed method is shown in sixth line. Since we did not predict it except the proposed method, we denote them by - . As for the proposed method, a restored image is provided by only one calculation whereas more than two times calculation were needed for the other methods. Accordingly, the calculation time of the compared methods were around twice the length of that of our proposing method. Note that 30 times of iteration were necessary for the Belge's method and the calculation time was more than one hour. In contrast, the prediction time of our method is extremely short.

We compare the restored images of each method next. We show the original image $f^{[32]}$ in Fig. 3. A certain gray domain in the vicinity of the center of the image is a moving object. We show the degraded image $\boldsymbol{g}^{[32]}$ in Fig. 4. From the degraded image, distinction of the detail patterns in the original image is difficult. 

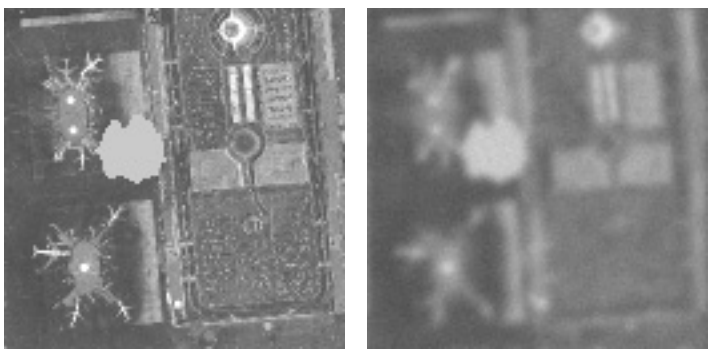

Figure 3: Original image $\left(f^{[32]}\right)$.

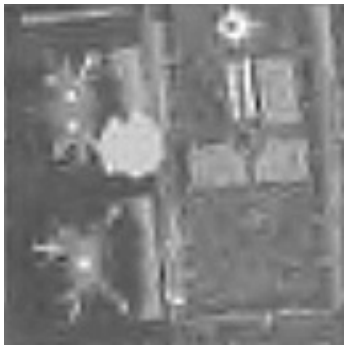

Figure 4: Degraded image $\left(g^{[32]}\right)$

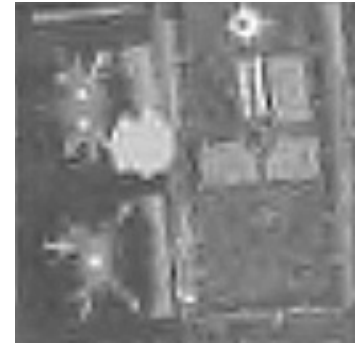

Figure 5: Restored image (proposed method).

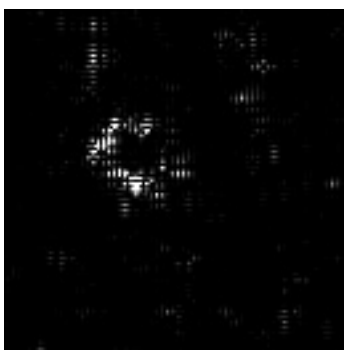

Figure 6: Restored image (compared method 1).

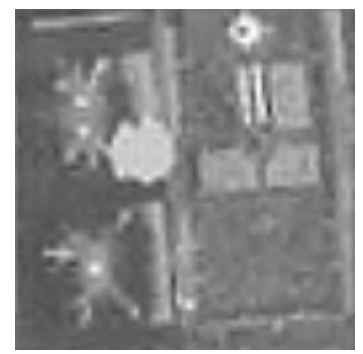

Figure 7: Squared error of Fig. 5 and Fig. 6.

Figure 8: Restored image (compared method 2).

We show the restored image $\boldsymbol{f}_{\text {rest }}^{[32]}$ by our method in Fig. 5. We can see that the edge in Fig. 5 is clearer than those in Fig. 4, and detailed patterns in Fig. 3 appear in the restored image to some extent. On the other hand, we show the restored image of the compared method 1 in Fig. 6. Comparing Fig. 6 with Fig. 5, they are almost distinguishable subjectively. In addition, we show the squared error of Figs. 6 and 5 in Fig. 7. The squared error images express that it is black when the error is 0 and it is close to white as the error is big. The following squared error images are displayed with the same scale. In Fig. 7, most of the errors are seen only around the edge of a foreground image, and we can understand that Fig. 6 is comparatively close to Fig. 5. Furthermore, we show the restored image of the compared method 2 in Fig. 8 and the squared error of Figs. 5 and 8 in Fig. 9. Fig. 8 is
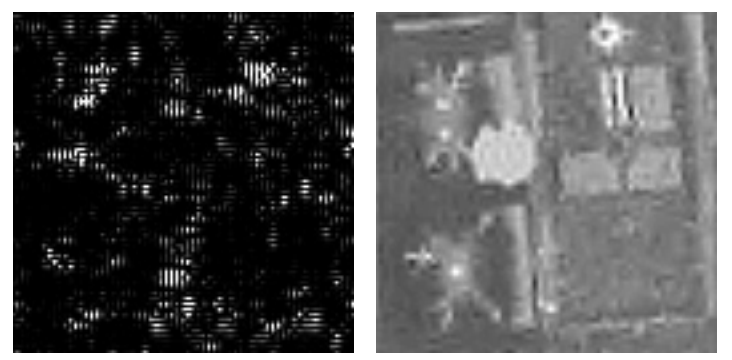

Figure 9: Squared error of Fig. 5 and Fig. 8.

Figure 10: Restored image (Belge et al.'s method).
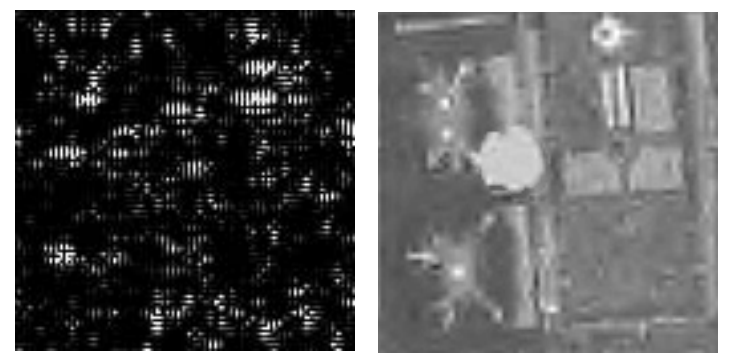

Figure 11: Squared error of Fig. 5 and Fig. 10.

Figure 12: Predicted image $\left(f_{\text {pred }}^{[32]}\right)$.

rather inferior in sharpness of the detailed patterns to Fig. 5. Since the degraded image was used for the initial image in the compared method 2, it is thought that the degradation factors were not removed, though the cost value was the same level as the proposed method. It proves this point in Fig. 9 that the comparatively large errors are seen around the edges. Moreover, we show restored image of the method by Belge et al. in Fig. 10 and the squared error image of Figs. 5 and 10 in Fig. 11. As pointed before, although ISNR is small in this method, the detailed patterns are clear generally in Fig. 10. Fig. 11 shows that there is an error in each place, yet large difference is hardly recognized by the subjective comparison between Figs. 5 and 10 .

In addition, we show predicted image $f_{\text {pred }}^{[32]}$ in Fig. 12. Except for the certain prediction errors which appears around the moving object, almost correct image is obtained.

\subsubsection{Evaluation of Simulation Result for a Moving Image}

We evaluate the restored images in each frame quantitatively next. We show the cost value of the restored moving image for each frame in Fig. 13 and the predicted or the initial moving images in Fig. 14. We plotted the results of the proposed method in a solid line, the compared method 1 in a dashed line and the compared method 2 in a chain line. We also plot- 
Table 3: Comparison of each calculation time of restoration for 36 frame.

\begin{tabular}{c||c|c|c|c}
\hline \hline item name & Proposed & Compared 1 & Compared 2 & (Belge et al., 2000) \\
\hline Total iteration number of times & 36 & 71 & 71 & 1176 \\
Average iterative number of times per a frame & 1 & 1.97 & 1.97 & 32.7 \\
Total calculation time & $1: 36^{\prime} 41^{\prime \prime}$ & $3: 03^{\prime} 30^{\prime \prime}$ & $2: 57^{\prime} 38^{\prime \prime}$ & $46: 31^{\prime} 34^{\prime \prime}$ \\
Prediction time (within total calc. time) & $34^{\prime \prime}$ & - & - & - \\
\hline
\end{tabular}
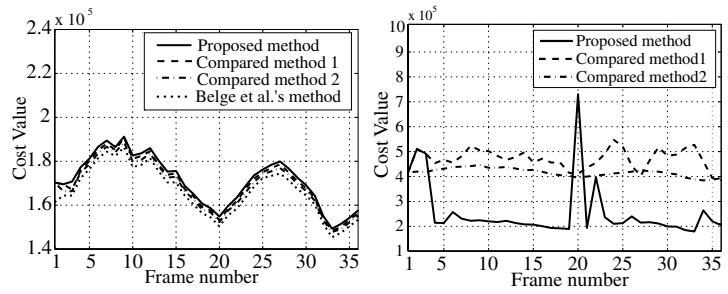

Figure 13: Cost value of the restored images.

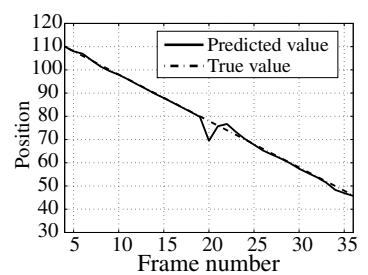

(a) Vertical direction $\bar{c}_{x}^{[k]}$
Figure 14: Cost value of the initial images.

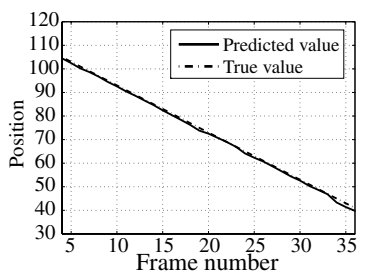

(b) Horizontal direction $\bar{c}_{y}^{[k]}$
Figure 15: Predicted value of the center of mass $\left(\overline{\boldsymbol{c}}^{[k]}\right)$.

ted the result of the Belge et al.'s method in a dotted line for reference. In Fig. 13, large differences are not recognized between each method. In addition, we show the position of center of gravity of the moving object in Fig. 15. Fig. 15(a) shows vertical direction and (b) shows horizontal direction, respectively. We plotted the true value in a chain line. The horizontal axis begins with $k=4$ since we did not predict until $k=3$. In Fig. 14, the cost value of the predicted image in our method is almost half of that of the initial value in other methods in most frames, which the similar tendency has seen in Table 2. The reason that the cost values in $1 \mathrm{st}-3 \mathrm{rd}, 20$ th and 22 nd frames are large is thought as follows: for the 1st-3rd frame, the previous restored image is used for an initial image directly. As for 20th and 22nd frame, we can see an larger error in a center of gravity prediction in Fig. 15(a) around these frame, so it is thought that the cost value increased by influence around the moving object domain that took the wrong prediction. However, since the domain except the moving object domain in the predicted image of these frames is predicted correctly, the cost value of the restored image of the proposed method decreased enough by one calculation.
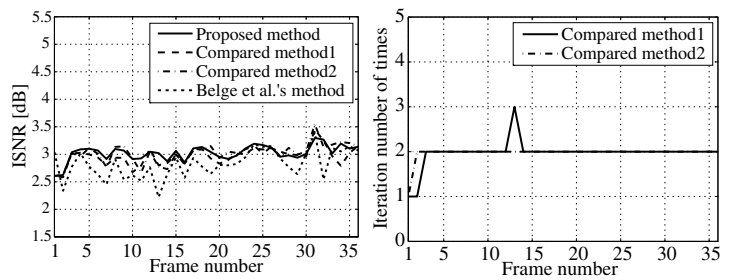

Figure 16: ISNR of each method.

Figure 17: Iteration number of times of the compared methods.

From Fig. 15(a) and (b), the center of gravity is approximately correctly predicted in the other frames.

In addition, we show ISNR of the unknown original image and the restored image in each frame by each method in Fig. 16. ISNR of proposed method is approximately the same as the compared method 1 and 2. However, ISNR of the Belge et al.'s method is smaller than the other methods in all frames. Therefore, we understand that there is a similar tendency to the above 32nd frame in the other frame.

Furthermore, we show the iterative calculation number of times in the compared methods 1 and 2 in Fig. 17. The iteration number of times of compared methods were two or three. In Table 3, we show the total iteration number of times and calculation time for the restoration processing of 36 frames. We also show the prediction time among the calculation time in the fourth line. The total iteration number of times of the proposed method were about half of the compared methods 1 and 2, and the similar tendency is seen about the calculation time. The average prediction time in the proposed method was 1 [sec] whereas the average time of one time iterative calculation was 150 [sec]. Therefore, it was shown that the prediction time in proposed method is extremely smaller than that of the iterative calculation.

\subsubsection{Summary of Simulations}

The restoration result of our proposed method has approximately the same precision quantitatively and took about half calculation time compared to the compared method 1 and 2. From the subjective evaluation, the restored images of proposed method have same or 


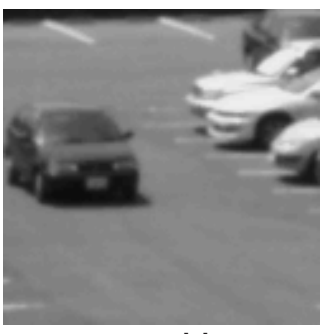

(a) $g^{[5]}$

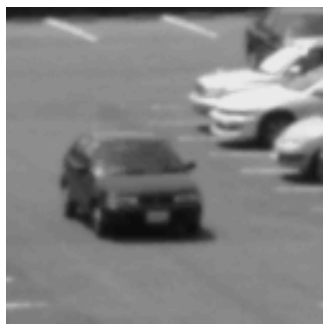

(b) $\boldsymbol{g}^{[40]}$

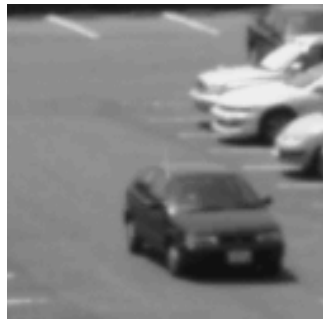

(c) $g^{[75]}$

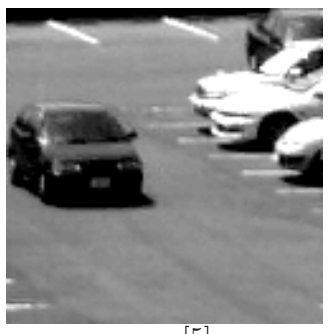

$\left(\mathrm{a}^{\prime}\right) \boldsymbol{f}_{\text {rest }}^{[5]}$

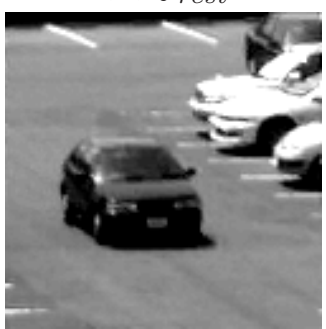

$\left(\mathrm{b}^{\prime}\right) \boldsymbol{f}_{\text {rest }}^{[40]}$

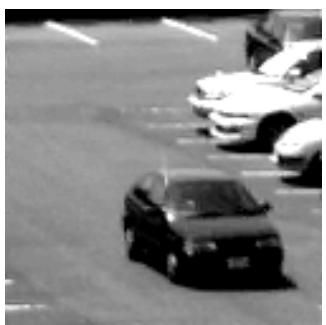

$\left(\mathrm{c}^{\prime}\right) \boldsymbol{f}_{\text {rest }}^{[75]}$
Figure 18: Restoration results of the real moving image (5th, 40th and 75th frame).

even better quality than those of the compared methods. In addition, the large difference was hardly found by the subjective comparison with the Belge et al.'s restoration result. Therefore, our result has sufficient quality enough in addition to the reduction of computation time. It is concluded that the proposed method has the effectiveness in the artificially generated degraded moving image restoration by quantitative and qualitative evaluation.

\subsection{Experiment for a Real Moving Image}

We show the restoration result using a real original image. We took a $128 \times 128$ pixel moving image of 256 gradation by an optically blurred fixed CCD video camera. Since this moving image does not include motion blur, we applied the degradation model (8) and executed restoration calculation. The actual degradation parameters in the moving image are unknown, but we set as follows experimentally. As for the optical blur, we supposed a Gauss function of the fixed variance $\sigma^{2}=1.0$. As for the disturbance $\boldsymbol{u}^{[k]}$, we supposed to be a Gaussian noise of SN ratio of 30dB. In Fig. 18, we show degraded images and restored images of 5th, 40th and 75th frame. Each image in the right column shows the restored image $\boldsymbol{f}_{\text {rest }}^{[k]}$ corresponding to the degraded image $\boldsymbol{g}^{[k]}$ in the left column. From the experimental result, we can see that the edges of the moving vehicle and parking cars are clear compared to its degraded image. Therefore, we can make sure that the restored images have good quality by applying above degradation model.

\section{CONCLUSION}

This paper developed the effective restoration method for degraded moving image. The dynamics of the moving image is modeled and a novel calculation algorithm is proposed. From the computer simulation of the artificially generated moving image, the calculation time was shortened and performances are increased quantitatively and qualitatively compared with other methods. Furthermore, we performed restoration calculation for a real moving image, which also show a good result, qualitatively.

Although in simulations and experiment, all considered degradation of the matrix $\boldsymbol{H}$ was optical blur, it can cope with various degradation factors. In addition, we can cope with other kinds object motion by changing the transition matrix $T(\cdot)$. Therefore, our proposed method can apply to various restoration processing.

\section{REFERENCES}

Banham, M. and Katsaggelos, A. (1997). Digital image restoration. IEEE Signal Process. Mag., 14(2):24-41.

Belge, M., Kilmer, M., and Miller, E. (2000). Wavelet domain image restoration with adaptive edge-preserving regularization. IEEE Trans. on. Image Processing, 9(4):597-608.

Charbonnier, P., Blanc-Feraud, L., Aubert, G., and Barlaud, M. (1997). Deterministic edge-preserving regularization in computed imaging. IEEE Trans. on. Image Processing, 6(2):298-311.

Daubechies, I. (1992). Ten Lectures on Wavelets. SIAM.

Geman, D. and Yang, C. (1995). Nonlinear image recovery with half-quadratic regularization. IEEE Trans. on Image Processing, 4(7):932-946.

Mallat, S. (1989). A theory for multiresolution signal decomposition: the wavelet representation. IEEE Trans. on. PAMI, 11(7):674-693.

Osher, S., Ruden, L., and Fatemi, E. (1992). Nonlinear total variation based noise removal algorithms. Phis. D, 60:259-268. 